\title{
Impulse Variables, Vortex Dipoles and Applications
}

\author{
Ricardo Cortez* \\ University of California and Lawrence Berkeley Laboratory \\ Berkeley, CA 94720, USA
}

\begin{abstract}
A Lagrangian numerical method based on impulse variables is analyzed. The relation between impulse vectors and vortex dipoles with a prescribed dipole moment is presented and used to adapt the high-accuracy cutoff functions of vortex methods for use in impulse-based methods. The long-time implementation of the impulse method is shown to contain a source of error whose growth in time renders impulse methods impractical for use in interior flows. Applications for which impulse methods are well suited are identified.
\end{abstract}

\section{Introduction}

The Navier-Stokes equations are typically written in terms of the fluid velocity or vorticity. Most numerical methods are discretizations of either formulation of these equations. The recent introduction of a new variable, which has been called magnetization [3, 5], velicity [4], impetus [10], and impulse [9], led to a new formulation of the fluid equations [4, 12]. In terms of the new variable, the Euler equations display readily the Hamiltonian structure of fluid flow in any space dimension. Buttke [4] presented a Lagrangian numerical method based on discretizations of the impulse equations. These discretizations lead to systems which, in the absence of viscosity, conserve many invariants associated with the Hamiltonian. However, many questions regarding the method have been unresolved. For instance, specific flows for which the method is particularly useful have not been identified, the accuracy of impulse-based methods has not been studied for long-time simulations, convergence properties of the method and how to choose the cutoff functions were unknown. These questions are answered here. We present an argument that shows that the impulse blob discretizations used in [4] induce the same flow as smoothed vortex dipoles. Consequently the particle locations obtained with the impulse method approximate the locations obtained with a vortex method which uses the same smoothing parameters. This result allows one to import the role of the cutoff function used in vortex methods into impulse methods and validates the use of such functions in the present context.

We also show that the impulse flow is an approximation to the flow induced by vortex sheets and that in the impulse method as presented in [4], this approximation can lose accuracy in time as the vortex sheets stretch. The stretching of the vortex sheets is directly related to the growth in the magnitude of the impulse vectors defined on the sheets. A consequence of this result is that accurate long-time implementations of the method in some interior flows are not possible without further modifications of the method. However, there are applications for which impulse methods are very useful. For instance, problems which make use of vortex dipoles along free surfaces can be modeled with impulse variables since these represent dipoles and the impulse equations update the

${ }^{*}$ current address: Courant Institute, 251 Mercer Street, New York, NY 10012. 
dipole strengths appropriately. This was done in [9] to model the motion of an elastic membrane surrounding an incompressible fluid. Other applications of impulse methods include flow near solid boundaries. Summers and Chorin [16] have used a combination of impulse and vortex elements to model viscous flow past a sphere.

This paper begins with a brief presentation of the equations of motion for impulse. In Section 3 we show the connection between impulse and vortex dipoles and the relevance of this connection on the accuracy of the impulse method in long-time implementations. A numerical example that illustrates the results is presented in Section 4. A discussion of applications of impulse methods which do not present accuracy problems and for which impulse methods seem particularly attractive is presented in the concluding section.

\section{Equations of motion}

Let $\mathbf{u}$ be the velocity of an incompressible fluid. Impulse can be introduced starting with the fluid velocity at time $t=0$ and making a change of variables in the following way: let $\mathbf{m}$ be a vector field equivalent to $\mathbf{u}$ up to an arbitrary gradient; that is,

$$
\mathbf{m}=\mathbf{u}+\nabla \phi
$$

Since $\nabla \cdot \mathbf{u}=0$, Eq. 1 implies that $\mathbf{u}$ is the divergence-free part of the Hodge decomposition of $\mathbf{m}$ in $\mathbb{R}^{\mathrm{n}}$ (see [7]). This fact will be written symbolically as $\mathbf{u}=\mathbb{P} \mathbf{m}$. In two dimensions it is known that $\phi$ can be chosen in such a way that $\mathbf{m}$ has compact support only when the net vorticity is zero. The equations of motion for $\mathbf{m}$ in free space of any dimension are (see e.g. [4, 5, 9])

$$
\frac{D \mathbf{m}}{D t}=-(\nabla \mathbf{u})^{T} \mathbf{m}+\nu \Delta \mathbf{m}, \quad \mathbb{P} \mathbf{m}=\mathbf{u},
$$

where $D / D t=\partial / \partial t+\mathbf{u} \cdot \nabla$ is the derivative along particle trajectories, $\nabla \mathbf{u}$ is a matrix with entries $(\nabla \mathbf{u})_{i j}=\partial u_{i} / \partial x_{j}$, and $T$ denotes a transpose. For a derivation of Eq. 2 refer to [4, 9]. By substituting Eq. 1 into Eq. 2 we can check that the latter is consistent with the Navier-Stokes equations. In the remainder of this paper we consider Euler flow and so we set $\nu=0$.

The Euler equations in terms of impulse are

$$
\frac{D \mathbf{m}}{D t}=-(\nabla \mathbf{u})^{T} \mathbf{m}, \quad \mathbb{P} \mathbf{m}=\mathbf{u} .
$$

Consider a 2D surface in $\mathbb{R}^{3}$ or a curve in $\mathbb{R}^{2}$ (e.g., the level set of an arbitrary function) with the property that the impulse field initially defined on it is perpendicular to the surface. It was shown in [8] that as this surface moves with the flow induced by the impulse, the impulse vectors will evolve in such a way that they remain perpendicular to the evolved surface. In [8], these surfaces which remain perpendicular to $\mathbf{m}$ during the evolution of the flow are referred to as $\mathbf{m}$-orthogonal surfaces.

During the evolution of the flow, the area of an $\mathbf{m}$-orthogonal surface may change even when the flow is incompressible since the flow can accommodate larger areas by contracting in the direction normal to the surface. The growth of the area of an $\mathbf{m}$-orthogonal surface is proportional to the local growth of the magnitude of $\mathbf{m}$. This result has been discussed in [5] and [6] by interpreting the flow induced by an impulse vector as that of a small vortex loop. This issue is key in the numerical method, where discrete impulse vectors carry elements of volume. Each volume element can be thought of as the area of a piece of an $\mathbf{m}$-orthogonal surface multiplied by a thickness, where the length of each vector is proportional to the area it carries. In Section 3 we will show that the 
accuracy of the method depends on the distribution of particles on the $\mathbf{m}$-orthogonal surfaces, and those surfaces in the interior of a flow can develop regions where particles are sparse, leading to a catastrophic loss of accuracy.

\section{The Lagrangian numerical method}

The equations of motion along fluid particles are:

$$
\begin{aligned}
\frac{d}{d t} \mathbf{x}(\alpha, t) & =\mathbf{u}(\mathbf{x}), & \mathbf{x}(\alpha, 0) & =\alpha \\
\frac{d}{d t} \mathbf{m}(\mathbf{x}, t) & =-(\nabla \mathbf{u})^{T} \mathbf{m}(\mathbf{x}, t), & \mathbf{m}(\alpha, 0) & =\text { given } .
\end{aligned}
$$

In [4], the impulse field is discretized initially by subdividing the domain into cells with the volume (or area) of the $j$-th cell equal to $V^{j}$, and by letting $\mathbf{m}^{j}=\mathbf{m}\left(\alpha^{j}\right) V^{j}$, where $\alpha^{j}$ is the center of the cell. Then an approximate impulse field is given by the sum

$$
\tilde{\mathbf{m}}(\mathbf{x})=\sum_{j=1}^{N} \mathbf{m}^{j}(t) f_{\delta}\left(\mathbf{x}-\mathbf{x}^{j}\right),
$$

where $N$ is the total number of cells and $f_{\delta}$ is a smooth approximation of a Dirac delta function. The smoothing functions used in [4] were taken from vortex methods, however no discussion about

this choice was presented. More recently there has been some evidence that indicates that cutoff functions from vortex methods are in fact the appropriate choice [9]. In the next section we show that the choice of cutoff in Eq. 6 is associated with the same choice of cutoff function in a vortex method.

If we assume that the cutoff function $f_{\delta}$ is radially symmetric, we find that in two dimensions the velocity at $\mathbf{x}$ is given by

$$
\mathbf{u}(\mathbf{x})=\frac{1}{2 \pi} \sum_{j=1}^{N} \mathbf{m}^{j}\left[\frac{r F^{\prime}(r)-F(r)}{r^{2}}\right]-\hat{\mathbf{x}}^{j}\left(\mathbf{m}^{j} \cdot \hat{\mathbf{x}}^{j}\right)\left[\frac{r F^{\prime}(r)-2 F(r)}{r^{2}}\right],
$$

where $\hat{\mathbf{x}}^{j}=\left(\mathbf{x}-\mathbf{x}^{j}\right) / r$ and $r=\left|\mathbf{x}-\mathbf{x}^{j}\right|$ and $F(r)=\int_{|\mathbf{x}| \leq r} f_{\delta}(|\mathbf{x}|) d \mathbf{x}$ is called the shape factor, and depends only on the cutoff function. The impulse vectors $\mathbf{m}^{j}$ evolve with an equation that approximates Eq. 5, and is found by differentiation of the velocity expression above.

\subsection{Discrete impulse and vortex blobs in $\mathbb{R}^{2}$}

Suppose there is a single impulse blob in $\mathbb{R}^{2}$ located at the origin. One can show that the velocity at an arbitrary point $\mathbf{x}$ due to the impulse can be written as [8]

$$
\mathbf{u}(\mathbf{x})=\nabla \times\left(K * f_{\delta}\right) \mathbf{m}=K *\left(\nabla \times \mathbf{m} f_{\delta}\right),
$$

where $K(x, y)=(-y, x) /\left(x^{2}+y^{2}\right)$ is the vortex kernel. Equation 8 is the velocity field due to a vorticity distribution $\xi(\mathbf{x})=\nabla \times \mathbf{m} f_{\delta}(\mathbf{x})$. It is therefore important to analyze in detail the vorticity induced by impulse vectors in order to establish connections with vortex methods.

The vorticity induced by an approximate impulse field consisting of one blob, $\tilde{\mathbf{m}}(\mathbf{x})=\mathbf{m}(t) f_{\delta}(\mathbf{x})$, located at the origin is $\xi(\mathbf{x})=\nabla \times \mathbf{m} f_{\delta}(\mathbf{x})=\nabla f_{\delta}(\mathbf{x}) \times \mathbf{m}$. This implies that for $\xi=(0,0, \omega)^{T}$ we have

$$
\omega(\mathbf{x})=\nabla f_{\delta}(\mathbf{x}) \cdot(\mathbf{m} \times \hat{\mathbf{z}})
$$


where $\hat{\mathbf{z}}$ is the unit vector normal to the plane. The vector $(\mathbf{m} \times \hat{\mathbf{z}})$ has magnitude $|\mathbf{m}|$ and is perpendicular to $\mathbf{m}$. Thus, one can interpret the vorticity as $|\mathbf{m}|$ times the derivative of the cutoff function $f_{\delta}$ in the direction of $(\mathbf{m} \times \hat{\mathbf{z}})$. Let $\mathbf{h}$ represent a vector in the direction of $(\mathbf{m} \times \hat{\mathbf{z}})$ and let $|\mathbf{h}|=h$. Then, the vorticity may be written as

$$
\omega(\mathbf{x})=\lim _{h \rightarrow 0} \omega\left[f_{\delta}(\mathbf{x}+\mathbf{h})-f_{\delta}(\mathbf{x}-\mathbf{h})\right]
$$

where $\omega=|\mathbf{m}| / 2 h$. This expression shows explicitly that the vorticity induced by the discrete impulse is that of vortex dipoles (see Fig. 1). A vortex dipole is the limit of two vortex blobs of equal and opposite strength as their separation goes to zero; this limit is taken in a way that maintains a constant dipole moment equal to $-2 \mathbf{h} \omega=(\hat{\mathbf{z}} \times \mathbf{m})$.

One of our main conclusions is that the impulse discretization in Eq. 6 is equivalent to a distribution of dipoles along $\mathbf{m}$-orthogonal surfaces. Therefore the $\mathbf{m}$-orthogonal surfaces are vortex sheets as long as the impulse vectors are distributed densely along these surfaces; otherwise, the impulse field represents scattered vortex dipoles. To a given accuracy, the limit in Eq. 9 can be approximated by placing the two vortices a small distance apart. The resulting vorticity is that of a vortex method which uses the same cutoff function as the impulse discretization. This shows that the cutoff functions from vortex methods are in fact the appropriate blobs for the impulse method and that the relations between $h$ and the cutoff radius $\delta$ required in vortex methods must be respected in the present context too. It is also clear that for a small value of $h$, the particle locations given by the impulse method must approximate those obtained with a vortex method which uses the same cutoff function and radius. Our numerical experiments demonstrate this result.

We turn now to a discussion of the mechanism that causes the impulse method as presented in [4] to lose accuracy in time. We present the analysis in terms of the induced velocity field. If a vortex sheet has net vorticity zero, then the velocity at an arbitrary point due to this vorticity can be written as an line integral along the curve and the velocity due to impulse vectors which represent the same flow is a discretization of this integral.

\subsection{The velocity field}

Consider a finite vortex sheet with parametric representation $\mathbf{x}(s)$, where the arclength parameter $s$ is zero at the left end of the curve and $s=1$ at the right end. Assume that the net vorticity is zero and define $\Gamma(s)=\int_{0}^{s} \omega(\mathbf{x}(q)) d q$. From this definition it is clear that $\Gamma(0)=0$ and $\Gamma(1)=$ $\int_{0}^{1} \omega(\mathbf{x}(q)) d q=0$ by assumption. With the vortex method the velocity field is computed by using a smoothed vortex kernel of the type $K_{\delta}(x, y)=K(x, y) F(r)$, where $F(r)$ is the shape factor defined at the beginning of Section 3. Then the velocity field is approximated by $\mathbf{u}_{\delta}=K_{\delta} * \omega$. In the case at hand,

$$
\mathbf{u}_{\delta}(\mathbf{x})=\int_{0}^{1} \omega(\mathbf{x}(s)) K_{\delta}(\mathbf{x}-\mathbf{x}(s)) d s=\int_{0}^{1}\left[\frac{d}{d s} \Gamma(s)\right] K_{\delta}(\mathbf{x}-\mathbf{x}(s)) d s .
$$

One can integrate by parts to obtain

$$
\mathbf{u}_{\delta}(\mathbf{x})=-\int_{0}^{1} \Gamma(s)\left[\frac{d}{d s} K_{\delta}(\mathbf{x}-\mathbf{x}(s))\right] d s .
$$

Note that $\frac{d}{d s} K_{\delta}(\mathbf{x}-\mathbf{x}(s))=-\left(\nabla K_{\delta}\right) \mathbf{x}^{\prime}(s)$, where $\mathbf{x}^{\prime}(s)$ is the unit vector tangent to the curve. Upon calculating $\left(\nabla K_{\delta}\right)$, we find that

$$
2 \pi \nabla K_{\delta}(\mathbf{x}-\mathbf{x}(s)) \mathbf{x}^{\prime}(s)=-\left(\hat{\mathbf{x}}(s) \cdot\left(\hat{\mathbf{z}} \times \mathbf{x}^{\prime}\right)\right) \hat{\mathbf{x}}(s)\left[\frac{r F^{\prime}-2 F}{r^{2}}\right]+\left(\hat{\mathbf{z}} \times \mathbf{x}^{\prime}(s)\right)\left[\frac{r F^{\prime}-F}{r^{2}}\right],
$$


where $r=|\mathbf{x}-\mathbf{x}(s)|$ and $\hat{\mathbf{x}}(s)=(\mathbf{x}-\mathbf{x}(s)) / r$. Therefore

$$
\mathbf{u}_{\delta}(\mathbf{x})=\frac{1}{2 \pi} \int_{0}^{1} \mathbf{m}(s)\left[\frac{r F^{\prime}-F}{r^{2}}\right]-(\hat{\mathbf{x}}(s) \cdot \mathbf{m}(s)) \hat{\mathbf{x}}(s)\left[\frac{r F^{\prime}-2 F}{r^{2}}\right] d s,
$$

for $\mathbf{m}(s)=\Gamma(s)\left(\hat{\mathbf{z}} \times \mathbf{x}^{\prime}(s)\right)$. Equation 7 is a discretization of this integral. Note that $\mathbf{m}(s)$ is normal to the vortex sheet.

At $t=0$ the impulse discretization can be arranged along the vortex sheet in such a way that Eq. 7 may represent a standard quadrature rule approximating the integral in Eq. 11. As the flow evolves the vortex sheet will stretch and wrap around itself [15], creating regions where the particles carrying impulse are far apart from their neighbors. This mechanism causes the flow to be one induced by a collection of vortex dipoles sparsely placed along the sheet and not the flow induced by a good approximation of the vortex sheet.

In practice, one discretizes impulse by identifying many sheets in which the impulse vectors can be defined. For example consider the problem of two vortex patches of constant vorticity $+\omega$ and $-\omega$ as shown in Fig. 2. Impulse vectors can be defined along horizontal sheets as shown in the figure. If $\mathbf{m}=\left(0, m_{2}\right)$, then $m_{2}(x, y)=\int^{x} \omega(\zeta, y) d \zeta$.

\section{Numerical experiment}

Consider two circular vortex patches of radius 0.25 , strengths +1 and -1 , and centered at $(-0.5,0)$ and $(0.5,0)$ respectively. The patches were discretized with vortex blobs and this discretization was approximated by impulse vectors. In the impulse method, the vectors were placed along horizontal vortex sheets as in Fig. 2. Each sheet was divided into $N$ intervals of length $h=1 / N$, and the initial impulse vectors were placed at the center of each interval. This way, the velocity induced by the impulse represents the midpoint rule approximation of the velocity field obtained with a vortex method which uses the same cutoff function. The parameters used were $\delta=0.5$ and the initial inter-particle spacing $h=0.05$. As the flow evolves and the vortex sheets wrap around the vortex patches, the impulse vectors become sparsely distributed along the sheets and one can expect the midpoint rule along such an integration path to experience accuracy loss. The solution was found first with the appropriate vortex method and then solved using the impulse method. Both methods used the 2nd-order cutoff function $f_{\delta}(r)=\delta^{-2} f_{1}(r / \delta)$, where $f_{1}(r)=\frac{1}{2 \pi}\left(e^{-r^{2}}+\frac{1}{2} e^{-r^{2} / 2}\right)$. The particles were evolved with the impulse method to time $t=4.76$ and the positions of the vortices were compared with the ones obtained with the vortex method. Figure 3 shows the location of the vortices (circles) and impulse vectors (dots) given by the impulse method at $t=4.76$ as well as the locations of the vortices obtained with the vortex method. The vortex positions given by the vortex method indicate that the patches should still be approximately circular, with centers about 0.5 units higher than the locations given by the impulse method. The patches found with the impulse method appear to be dismembering. It is clear that in long-time implementations, the impulse vectors eventually drift so far apart on the $\mathbf{m}$-orthogonal surfaces that they do not approximate the vortex flow; the impulse flow is that of vortex dipoles at the base of the vectors. We point out that even as the impulse method loses the accuracy with which it approximates the flow induced by the two vortices, the invariants associated with the Hamiltonian dipole system continue to be preserved to an accuracy of $10^{-8}$.

The accuracy in the impulse approximation of the velocity field given by the vortex method can be maintained by adding new impulse vectors throughout the evolution of the flow. New vectors can be introduced whenever the separation between contiguous vectors surpasses a predetermined tolerance. This refinement is done only in the approximation of the vortex flow by impulse vectors, 
not to new vortex blob discretizations of the patches. The number of vortex blobs is fixed at the beginning of the problem. In the refinement procedure we consider the $\mathbf{m}$-orthogonal surfaces as piece-wise linear curves with a vector at the midpoint of each linear piece (see Fig. 4).

If a linear piece is too long (exceeds the tolerance), we replace that vector by two equally-spaced vectors in the same direction and with half the magnitude of the original one. This procedure is designed to preserve the discrete impulse, $\mathbf{I}=\sum \mathbf{m}^{j}$, exactly.

The results of the impulse method with refinement for the problem of two vortex patches are shown in Fig. 5 at time $t=4.72$. In this case, an impulse vector was replaced by two vectors whenever the discretization size (the inter-particle spacing) reached twice the initial value. In comparison with Fig. 3, it is clear that the refinement procedure allows the impulse method to maintain the accuracy with which it approximates the flow.

We computed the order of accuracy of the impulse method by approximating the same vortex sheets with twice as many impulse vectors as the previous discretization. The solution was carried to the same final time, which was chosen arbitrarily as representative of times when the original impulse method is known to yield wrong answers. At other final times, the results were similar. We define the error

$$
\text { Error }=\frac{1}{N} \sum_{j=1}^{N}\left|\mathbf{x}_{V}^{j}-\mathbf{x}_{I}^{j}\right|,
$$

where $\mathbf{x}_{V}^{j}$ is the location of the $j$-th particle obtained with the vortex method and $\mathbf{x}_{I}^{j}$ is the location obtained with the impulse method. Figure 6 shows that the errors in the vortex locations decreased by about a factor of 4 . This is an indication of the second order accuracy as one would expect from the midpoint-rule approximation of the vortex flow. In spite of the explicit dependence of the energy $1 / 2 \sum \mathbf{m}^{j} \cdot \mathbf{u}\left(\mathbf{x}^{j}\right)$ on the discretization, energy was conserved to within $1.5 \%$ of its original value in this computation. The refinement procedure allows the impulse to maintain the initial accuracy in its approximation of the vortex flow but it is clearly not a practical implementation of the impulse method. The number of particles continually increases without bound making the impulse method prohibitively expensive without the use of fast summation techniques. In Fig. 5 (at time 4.72) there are four times as many particles as there were at $t=0$.

The accuracy of the impulse discretization can also be maintained by exploiting the nonuniqueness of the impulse field. One can let the flow evolve for a short time, then define more convenient vortex sheets through the vortices along which a new impulse field can be defined. Then one can continue the evolution of the flow. The new vortex sheets are the new $\mathbf{m}$-orthogonal surfaces. Ideally, the new vortex sheets would weave through many vortices while remaining as straight and as short as possible. This would keep bounded the number of impulse vectors required to approximate the flow with the given accuracy.

The test problem of the vortex patches was solved using this technique. Figure 7 shows the particle positions obtained with this method at time $t=4.72$ as in the previous figures. In this case, the new $\mathbf{m}$-orthogonal curves are replaced at the end of every time step to ensure that the initial discretization defines the accuracy of the method. Table 4 shows the errors, defined in Eq. 12, from two impulse discretizations corresponding to inter-particle separations of $h=0.05$ and $h=0.025$. The numbers in the table indicate that the errors increase linearly in time and decrease by about a factor of four when the discretization is halved. This is the same result shown in Fig. 6 for the case of the impulse method with refinement. However, the number of particles using this resurfacing procedure does not increase without bound as it does in the refinement. For instance, the number of particles in this example (for $h=0.025$ ) was initially 590, while at later times it fluctuates about 1000. 
Table I: Errors for the vortex patch problem using the impulse method with resurfacing and two different discretizations. The method used cutoff parameter $\delta=0.5$ and time step $\Delta t=0.08 . \mathbf{x}_{V}^{j}$ is the position of the $j$-th particle obtained with the vortex method, $\mathbf{x}_{I}^{j}$ is the position of the same particle obtained with the impulse method.

\begin{tabular}{|c|c|c|}
\hline & $h=0.05$ & $h=0.025$ \\
\hline time & $\frac{1}{N} \sum_{1}^{N}\left|\mathbf{x}_{V}^{j}-\mathbf{x}_{I}^{j}\right|$ & $\frac{1}{N} \sum_{1}^{N}\left|\mathbf{x}_{V}^{j}-\mathbf{x}_{I}^{j}\right|$ \\
\hline \hline$t=2.4$ & $4.2806 \mathrm{e}-04$ & $1.1063 \mathrm{e}-04$ \\
\hline$t=4.72$ & $8.0132 \mathrm{e}-04$ & $2.0817 \mathrm{e}-04$ \\
\hline$t=7.92$ & $12.648 \mathrm{e}-04$ & $3.3106 \mathrm{e}-04$ \\
\hline
\end{tabular}

\section{Conclusions}

The connection between the velocity field due to vortex blobs and the velocity field induced by impulse blobs has been established. The connection is that the velocity field induced by a distribution of vortices on a sheet in two dimensions can be written as a line integral along the sheet. An impulse field which models the same flow is an approximation of this line integral. An extension of these arguments to three dimensions shows that a large closed loop of vorticity induces a velocity field that can be approximated by an integral over any surface that spans the loop (see $[6,8]$ ). When the integration path (the vortex sheet) is carried by the flow, the path stretches and the approximation of the line integral loses accuracy as the points along the path become sparse. The stretching of the path results in the growth of the magnitude of impulse vectors. It is clear that impulse methods can be less efficient than vortex methods when portions of the vortex sheets have zero vorticity, as in the example of the patches. The variants of the impulse method presented here support the results and allows long-time implementations of the method. However they do not seem to be practical for more general problems and they are not easily extended to three dimensions.

The interpretation of impulse vectors as vortex dipoles with prescribed dipole moment has been explained. The main consequences of this interpretation is that the high-accuracy cutoff functions of vortex methods are the correct cutoff functions for impulse methods, and that the role of the cutoffs in the accuracy analysis of vortex methods can be imported into impulse methods. The convergence of the particle locations given by the impulse method to the locations given by the corresponding vortex method (the one that uses the same cutoff parameters) was demonstrated numerically.

Problems that call for the use of vortex dipoles or ones in which vortex sheets do not suffer extensive stretching are clear candidates for the application of impulse methods. Vortex dipoles have been used to model free-surface motion [1,2] and to introduce surface forces effects [14]. Impulse variables provide important advantages in this type of application because the equations of motion for impulse correctly update the dipole strengths. Since forces are rates of change of impulse, the impulse evolution equations can easily be written to include forces. This was done in [9], where the motion of an elastic membrane surrounding an incompressible fluid was tracked. There, the surface forces were due to variable tension, as is the case in applications such as the blood flow in the heart cavities and other problems in physiology [11, 13].

The vorticity induced by the impulse approximation in Eq. 6 has the property of being solenoidal in any space dimension, which makes it attractive to use an impulse formulation for the creation 
of vorticity near solid boundaries [16]. However, as the new elements diffuse into the flow interior, they must be converted into vortex elements in order to avoid carrying out the computation while extensive stretching of the vortex sheets occurs.

\section{Acknowledgements}

This work was supported in part by the Applied Mathematical Sciences Subprogram of the Office of Energy Research, U.S. Department of Energy under Contract DE-AC03-76SF00098 and by the Air Force Office of Scientific Research AASERT Grant FDF49620-93-1-0053.

\section{References}

[1] Baker, G.R., Meiron, D.I. and Orszag, S.A., "Generalized Vortex Methods for Free Surface Flow Problems," J. Fluid Mech., 123, pp. 477-501, 1982.

[2] Baker, G.R., Meiron, D.I. and Orszag, S.A., "Generalized Vortex Methods for Free Surface Flow Problems II: Radiating Waves," J. Sci. Comp., 4, pp. 237-259, 1989.

[3] Buttke, T.F., "The Hamiltonian Structure of 3D Fluid Flow," Proc. of Int'l. Workshop on Vortex Flows and Related Numerical Methods, Grenoble, France, June 15-20 1992.

[4] Buttke, T.F., "Velicity Methods: Lagrangian Numerical Methods which Preserve the Hamiltonian Structure of Incompressible Fluid Flow," Proc. NATO Adv. Res. Workshop: Vortex Flows and Related Numerical Methods, Grenoble, France, edited by Beale, Cottet and Huberson, Kluwer, pp. 39-58, 1993.

[5] Buttke, T.F. and Chorin, A.J., "Turbulence Calculations in Magnetization Variables," Appl. Num. Math., 12, pp. 47-54, 1993.

[6] Chorin, A.J., Vortex Methods, Les Houches Summer School of Theoretical Physics, Les Houches, France, 1993.

[7] Chorin, A.J. and Marsden, J.E., A Mathematical Introduction to Fluid Mechanics, SpringerVerlag, New York, 1979.

[8] Cortez, R., Impulse-based Particle Methods for Fluid Flow, PhD thesis, University of California, Berkeley, LBL report \#37206, 1995.

[9] Cortez, R., "An Impulse-based Approximation of Fluid Motion due to Boundary Forces," $J$. Comp. Phys, 123, pp. 341-353, 1996.

[10] Maddocks, J.H. and Pego, R.L., "An Unconstrained Hamiltonian Formulation for Incompressible Fluid Flow," Comm. Math. Phys., 170, pp. 207-217, 1995.

[11] McCracken, M.F. and Peskin, C.S., "A Vortex Method for Blood Flow through Heart Valves," J. Comp. Phys, 35, pp. 183-205, 1980.

[12] Oseledets, V.I., "On a New Way of Writing the Navier-Stokes Equation: The Hamiltonian Formalism," Commun. Moscow Math. Society, 44, pp. 210-211, 1989.

[13] Peskin, C.S., "Numerical Analysis of Blood Flow in the Heart," J. Comp. Phys, 25, pp. 220$252,1977$. 
[14] Pullin, D.I., "Numerical Studies of Surface Tension Effects in Nonlinear Kelvin-Helmholtz and Rayleigh-Taylor Instability," J. Fluid Mech., 119, pp. 507-532, 1982.

[15] Saffman, P.G., Vortex Dynamics, Cambridge Univ. Press, 1992.

[16] Summers, D. and Chorin, A.J., "Hybrid Vortex/Magnet Methods for Flow over a Solid Boundary," in preparation. 


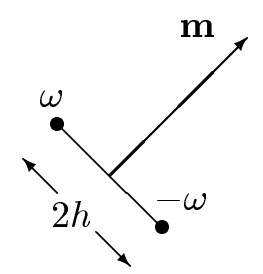

Figure 1: Vortex Pair Approximation to Impulse Vector.

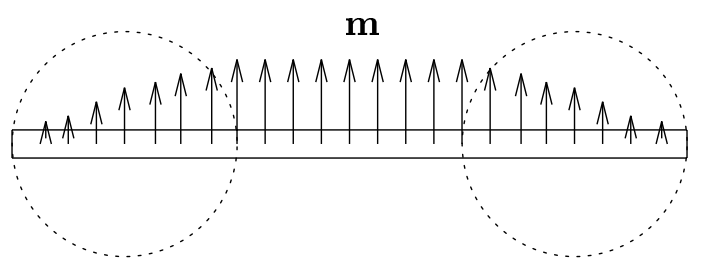

Figure 2: Two patches of constant vorticity and the corresponding impulse field defined on a horizontal sheet. 

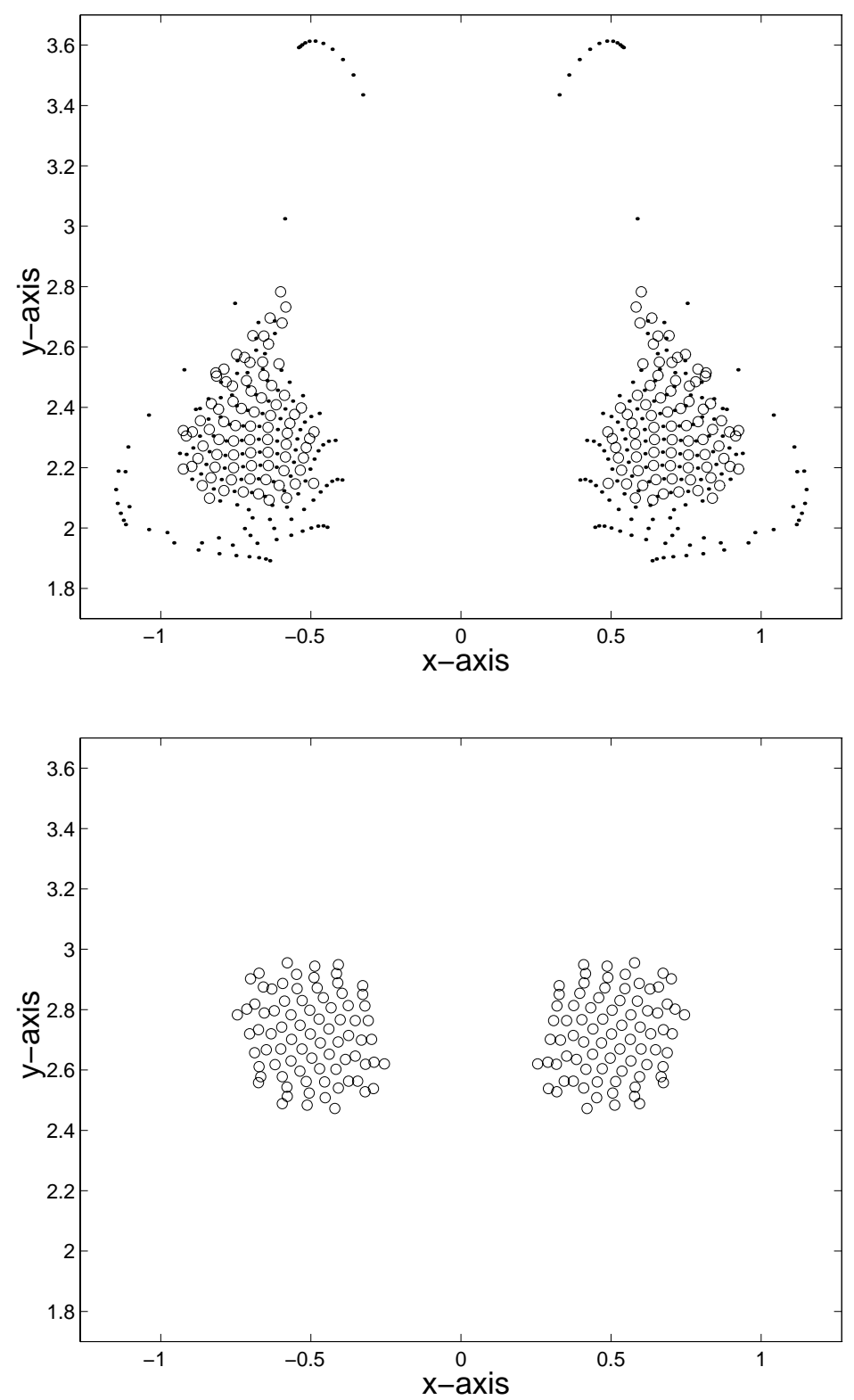

Figure 3: Solution at $t=4.76$. The top figure shows the vortex locations (circles) and the location of the impulse vectors (dots) obtained with the impulse method. The bottom figure shows the location of the vortices obtained with the vortex method.
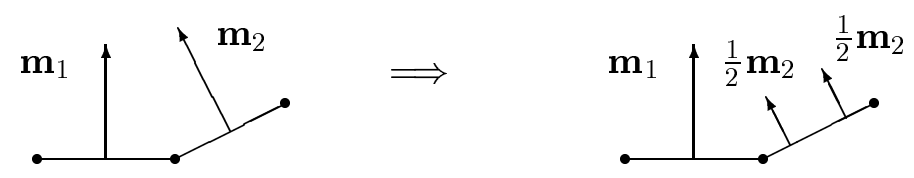

Figure 4: Refinement procedure which preserves impulse. 


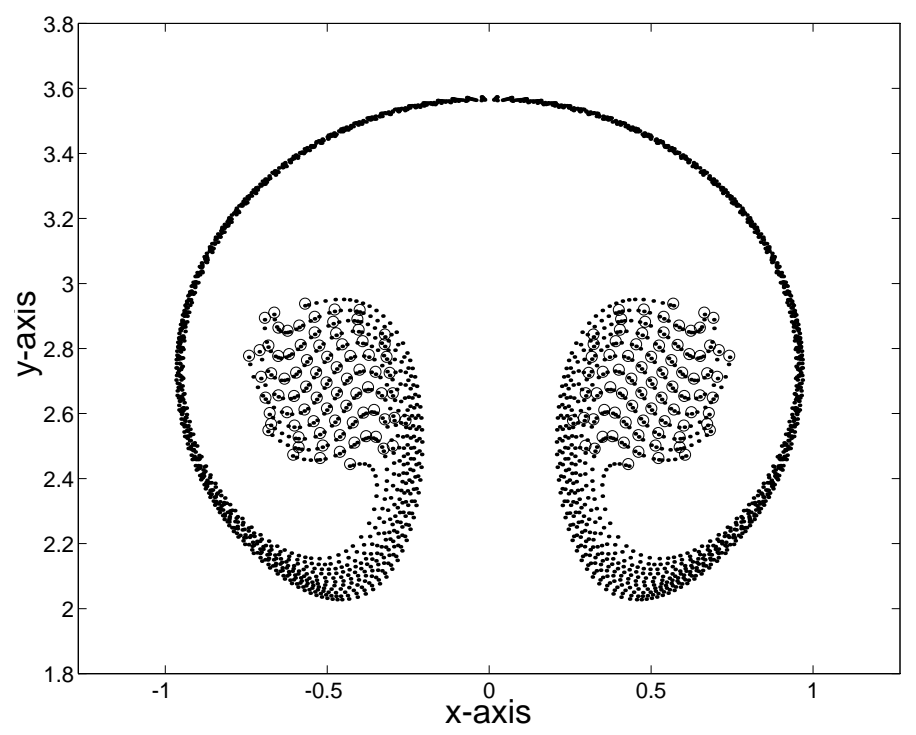

Figure 5: Solution at $t=4.72$ found with the impulse method with refinement.

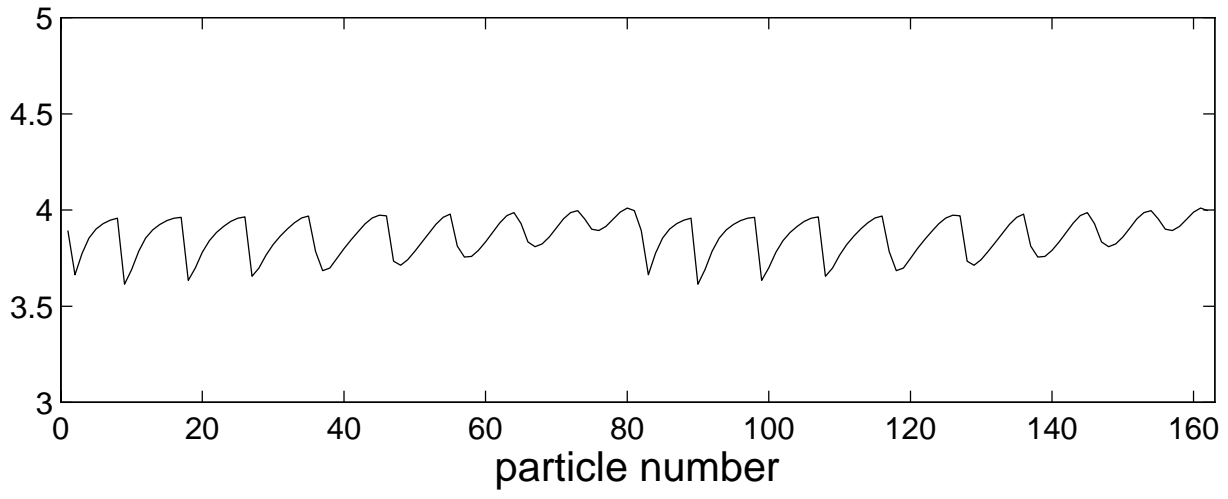

Figure 6: Comparison of vortex positions at $t=4.72$. The figure shows the ratio of the distances between corresponding vortices given by the impulse method with refinement and the vortex method, for $h=0.05$ and $h=0.025$. 


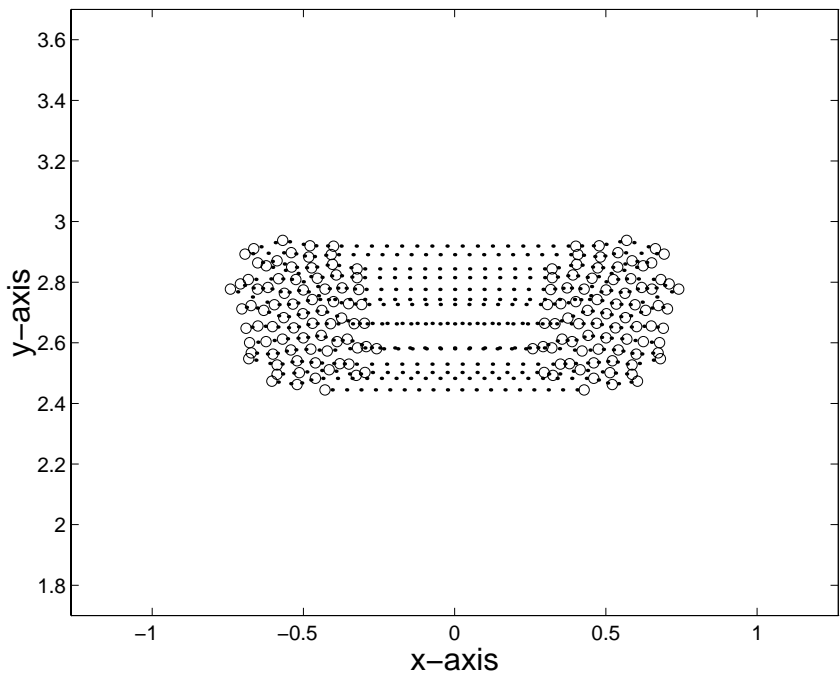

Figure 7: Solution at $t=4.72$ found with the impulse method with resurfacing with $\delta=0.5$ and $h=0.05$. 